\title{
DETERMINACIÓN DE RESISTENCIAANTIHELMÍNTICA (Fasciola hepatica) EN OVINOS FRENTE A ALBENDAZOL Y TRICLABENDAZOL, LA PAZ - BOLIVIA
}

\author{
Anthelminthic Resistance (F asciola hepatica) in Sheep Against Albendazole \\ and Triclabendazole, La PaZ - Bolivia
}

Willy Mamani L. ${ }^{1,2}$ y René Condori Q. ${ }^{3}$

\section{Resumin}

\begin{abstract}
Se determinó la resistencia de Fasciola hepatica frente a albendazol y triclabendazol en ovinos de dos rebaños en La Paz, Bolivia: uno cercano a Tambillo (rebaño 1) y otro cercano a Batallas (rebaño 2). Se eligió al azar 30 corderos de 8 a 9 meses de edad por rebaño, y se trataron con $10 \mathrm{mg} / \mathrm{kg}$ de albendazol ó $10 \mathrm{mg} / \mathrm{kg}$ de triclabendazol, quedando un grupo como control no tratado. Se midió la enzima gama glutamil transpeptidasa (GGT) y el número de huevos por gramo de heces (hpg) para la prueba de reducción de la oviposición. Los corderos se sacrificaron 8 semanas después del tratamiento y se contó el número de fasciolas. Se presentó una baja reducción de los niveles de GGT y de hpg en los grupos tratados con albendazol. En el grupo tratado con triclabendazol en el rebaño 1 se observó una de reducción de los niveles de GGT y hpg durante todo el estudio, no así en el rebaño 2. El porcentaje de reducción en los hígados fue de 13.6 y $2.3 \%$ para albendazol en los rebaños 1 y 2 , respectivamente, y de 98.0 y $36.3 \%$ para triclabendazol. Se concluye que existe resistencia de $F$. hepatica frente a albendazol en los dos rebaños y que la resistencia está en proceso de establecimiento para triclabendazol en el rebaño 2.
\end{abstract}

Palabras clave: resistencia antihelmíntica, albendazol, triclabendazol, ovinos

\section{AbStRact}

Fasciola hepatica resistance in sheep against albendazole and triclabendazole was evaluated in two farms in La Paz, Bolivia: one located near Tambillo (farm 1) and the other near Batallas (farm 2). Thirty male lambs of 7 to 8 months of age were selected in each farm. They were treated with $10 \mathrm{mg} / \mathrm{kg}$ of albendazole or $10 \mathrm{mg} / \mathrm{kg}$ of triclabendazole, and one group remained as non-treated. The gama glutamyl transpeptidase enzyme (GGT) profile and faecal eggs count reduction test (FECRT) were conducted. Lambs were slaughtered 8 weeks after treatment and the number of flukes in liver was counted. Minor reduction of GGT levels and faecal eggs count were observed in animals treated with albendazole. In the group treated with triclabendazole in farm 1 was observed a reduction

${ }^{1}$ Laboratorio de Parasitología Veterinaria, ${ }^{3}$ Laboratorio de Patología Clínica, Facultad de Medicina Veterinaria y Zootecnia, Universidad Pública y Autónoma de El Alto, La Paz, Bolivia

${ }^{2}$ E-mail: willymlmvzupea_2@hotmail.com 
of the GGT levels and faecal eggs count throughout the study, but not in farm 2 . The reduction of flukes in liver lambs was 13.6 and $2.3 \%$ for albendazol in farms 1 and 2 respectively, and 98.0 and $36.3 \%$ for triclabendazol. It was concluded that anthelminthic resistance of $F$. hepatica to albendazol occurred in both farms, while in farm 2 resistance was gradually appearing to triclabendazole.

Key words: anthelminthic resistance, albendazole, triclabendazole, sheep

\section{INTRODUCCIÓN}

El control eficiente de la fasciolosis en ovinos se puede lograr con un manejo adecuado de los campos de pastoreo y el uso estratégico y mínimo de antiparasitarios. Sin embargo, en la práctica se realiza la administración de antiparasitarios como una rutina sin control adecuado ni ningún criterio técnico, lo cual es la causa principal del aumento de la resistencia de los parásitos a los tratamientos.

Se considera que hay resistencia cuando la efectividad de un fármaco disminuye o cesa. Esto ocurre porque después de cada tratamiento sobrevive un pequeño número de individuos que son resistentes al fármaco utilizado, y éstos son los únicos que se reproducirán y contaminarán las pasturas con sus huevos (Jackson, 1993). El desarrollo de la resistencia antihelmíntica se logra por la continua selección de individuos (parásitos) resistentes, que se van desarrollando por el uso repetido de los antiparasitarios (Márquez, 2003), ya que se aumenta la frecuencia de genes de la resistencia en una población, hasta producir el reemplazo de la población sensible por una población resistente al fármaco con el consiguiente fracaso del tratamiento antihelmíntico (Romero et al., 1998). El establecimiento de una población resistente a un antihelmíntico es un proceso de carácter irreversible (Sievers y Alocilla, 2007).

Existen varios mecanismos moleculares por los cuales se presenta resistencia de $F$. hepatica al triclabendazol. Entre ellos se encuentra: a) la lenta reducción de la oxidación del triclabendazol (TCBZ) a triclabendazol sulfoxido (TCBZSO) que es el metabolito activo, b) la unión del TCBZ a proteínas no específicas restringe la habilidad del fármaco a unirse al sitio de acción, y c) el eflujo mediado por transportadores donde se sobreexpresa la acción de la glicoproteína-P (Pgp) permite la eliminación del fármaco desde las células (Márquez, 2003; Álvarez et al., 2007).

La resistencia antihelmíntica frente a la diversidad de fármacos se ha incrementado en los últimos años. La resistencia de parásitos de bovinos y ovinos a la ivermectina está reportada en Nueva Zelanda (West et al., 1994; Vermunt et al., 1995; Mason y McKay, 2006), Inglaterra (Stafford y Coles, 1999), Argentina (Fiel et al., 2000; Anziani et al., 2001; Suarez y Cristel, 2006), Nicaragua (Rimbaud et al., 2005) y en Chile (MoenenLocoz, 1998; Sievers y Fuentealba, 2003; Sievers y Alocilla, 2007). La resistencia de parásitos en ovinos al albendazol se ha reportado en Nueva Zelanda (Leathwick et al., 2006; Waghorn et al., 2006) y de F. hepatica al triclabendazol se ha reportado en Chile (Laverde, 2007), Australia (Overend y Bowen, 1995), y en los Países Bajos (Moll et al., 2000; Gaasenbeek et al., 2001).

El objetivo del presente trabajo fue determinar la resistencia de $F$. hepatica frente al albendazol y el triclabendazol en ovinos, mediante la prueba FECRT, niveles sanguíneos de GGT y la necropsia.

\section{Materiales y Métodos}

El estudio se realizó entre enero a abril de 2008 en dos rebaños del departamento de 
Cuadro 1. Promedio de Fasciola hepatica ( $\mathrm{n} \pm$ d.e.) recuperados de corderos machos de 1011 meses de edad, infectados naturalmente, y sacrificados en la semana 8 después del tratamiento antiparasitario, en dos rebaños de La Paz, Bolivia (2008)

\begin{tabular}{llccc}
\hline & Grupo & $\begin{array}{c}\text { F. hepatica } \\
\mathrm{n} \pm \text { d.e. }\end{array}$ & Rango & $\begin{array}{c}\text { Eficacia } \\
(\%)\end{array}$ \\
\hline Rebaño 1 & & & & \\
& Control & $75.0 \pm 16.0$ & $55-96$ & 13.6 \\
& Albendazol & $64.8 \pm 28.4$ & $26-115$ & 98.0 \\
& Triclabendazol & $1.5 \pm 2.4$ & $0-7$ & \\
Rebaño 2 & & & & 2.3 \\
& Control & $91.4 \pm 29.0$ & $53-137$ & 36.3 \\
\hline & Albendazol & $89.3 \pm 27.5$ & $54-130$ & \\
& Triclabendazol & $58.2 \pm 20.7$ & $28-85$ & \\
\hline
\end{tabular}

La Paz: el Rebaño 1, cercano a Tambillo donde se realiza una aplicación anual de antiparasitario, y el Rebaño 2, cercano a Batallas, con aplicación regular de tres dosis anuales de antiparasitario. En cada rebaño se eligió al azar a 30 ovinos machos de 8-9 meses de edad, de raza criolla, y se les distribuyó en tres grupos de 10 animales. Los animales no habían sido desparasitados en los 90 días previos al muestreo y se encontraban sobre praderas contaminadas naturalmente.

Los animales del Grupo 1 no fueron tratados (Control), los del Grupo 2 recibieron 10 $\mathrm{mg} / \mathrm{kg}$ de albendazol, vía oral, y los del Grupo 3, $10 \mathrm{mg}$ de triclabendazol, vía oral. Los animales permanecieron en sus praderas.

Se recolectó muestras fecales del recto la semana previa al tratamiento (at) y semanal por 8 semanas post tratamiento (pt). El recuento de huevos se hizo con la técnica cualitativa de sedimentación - flotación (Teuscher, 1965) y la técnica de sedimentación, expresándose los resultados en número de huevos por gramo de material fecal (hpg).
El porcentaje de reducción de la oviposición (FECRT) se calculó mediante la fórmula: $\mathrm{FECR}=\left(\mathrm{hpg}_{\mathrm{at}}-\mathrm{hpg}_{\mathrm{pt}}\right) \times 100 / \mathrm{hpg}_{\mathrm{at}}$ (Young et al., 1999). Además, se tomó muestras semanales de sangre para determinar los niveles de gama glutamil transpeptidasa (GGT).

Los corderos se sacrificaron a las ocho semanas del tratamiento. Se examinó los hígados y se hizo el contaje de Fasciola hepatica (Ross y O'Hagan, 1966). El porcentaje de eficacia fue calculado usando la siguiente fórmula:

Eficacia $(\%)=\left(\right.$ Media N ${ }^{\circ}$ de parásitos grupo control - Media N. ${ }^{\circ}$ de parásitos grupo tratado) $\mathrm{x}$ $100 /$ (Media N. ${ }^{\circ}$ de parásitos grupo control)

Los resultados se analizaron mediante un diseño factorial de 2 x 3 y se empleó la prueba de Tukey HSD para determinar diferencias entre grupos. Además, se usó estadística descriptiva. Los datos se analizaron con el paquete estadístico Statistix para Windows v 8.0. 

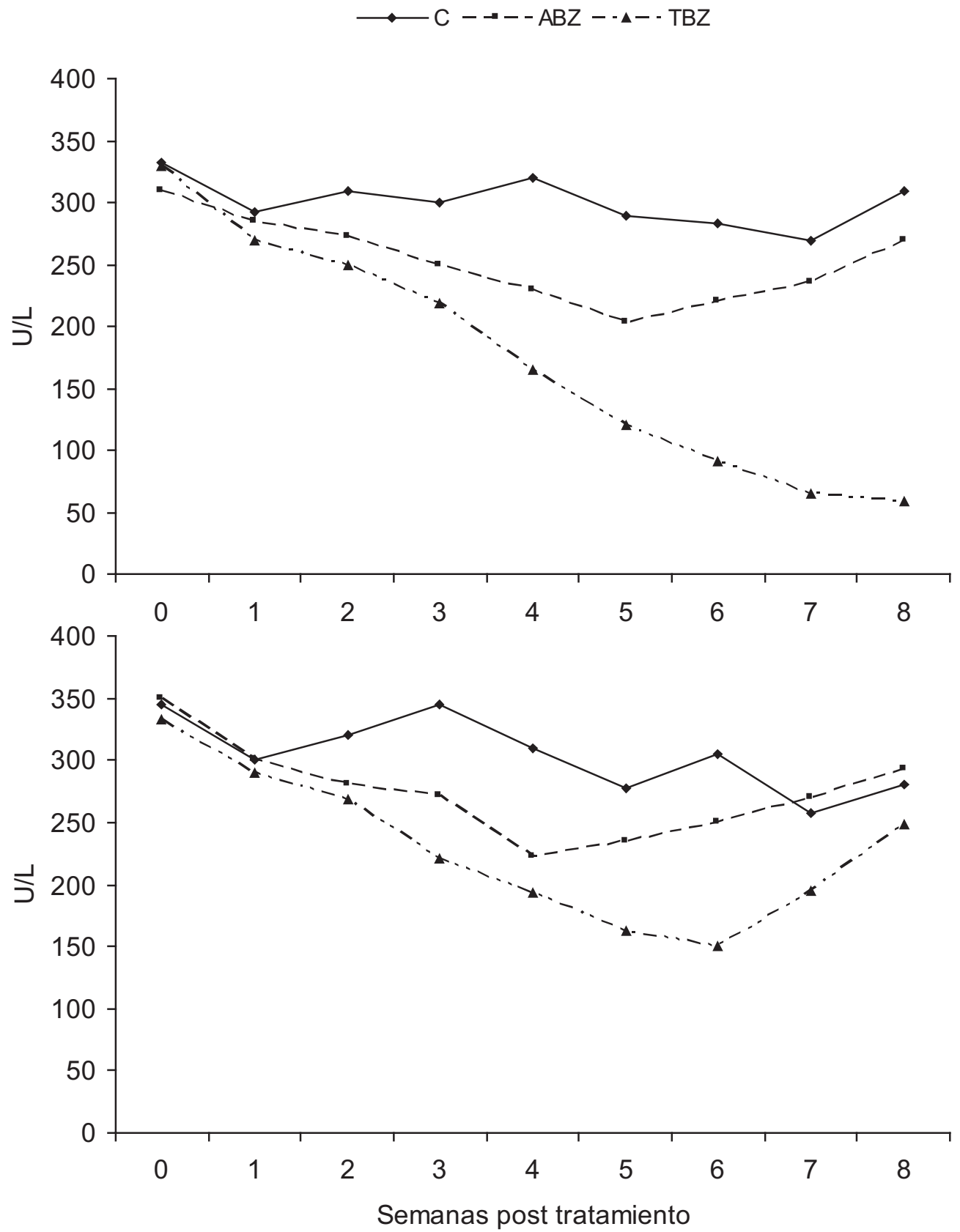

Figura 1. Perfil de la enzima gama glutamil transpeptidasa (GGT) expresada en unidades por litro (U/L), en muestras de sangre de los grupos de ovinos control (C), y tratados con albendazol (ABZ) y triclabendazol (TCBZ) en los rebaños 1 (panel superior) y 2 (panel inferior), durante ocho semanas post tratamiento en La Paz, Bolivia

\section{Resultados}

La enzima GGT se mantuvo elevada durante todo el estudio en el grupo Control en los dos rebaños. El grupo tratado con albendazol disminuyó hasta la cuarta semana post tratamiento $(\mathrm{p}<0.05)$ en el rebaño $2 \mathrm{y}$ hasta la quinta semana en el rebaño 1, para luego aumentar y tener los mismos niveles que el grupo control en la semana 7. En el grupo tratado con triclabendazol disminuyó 

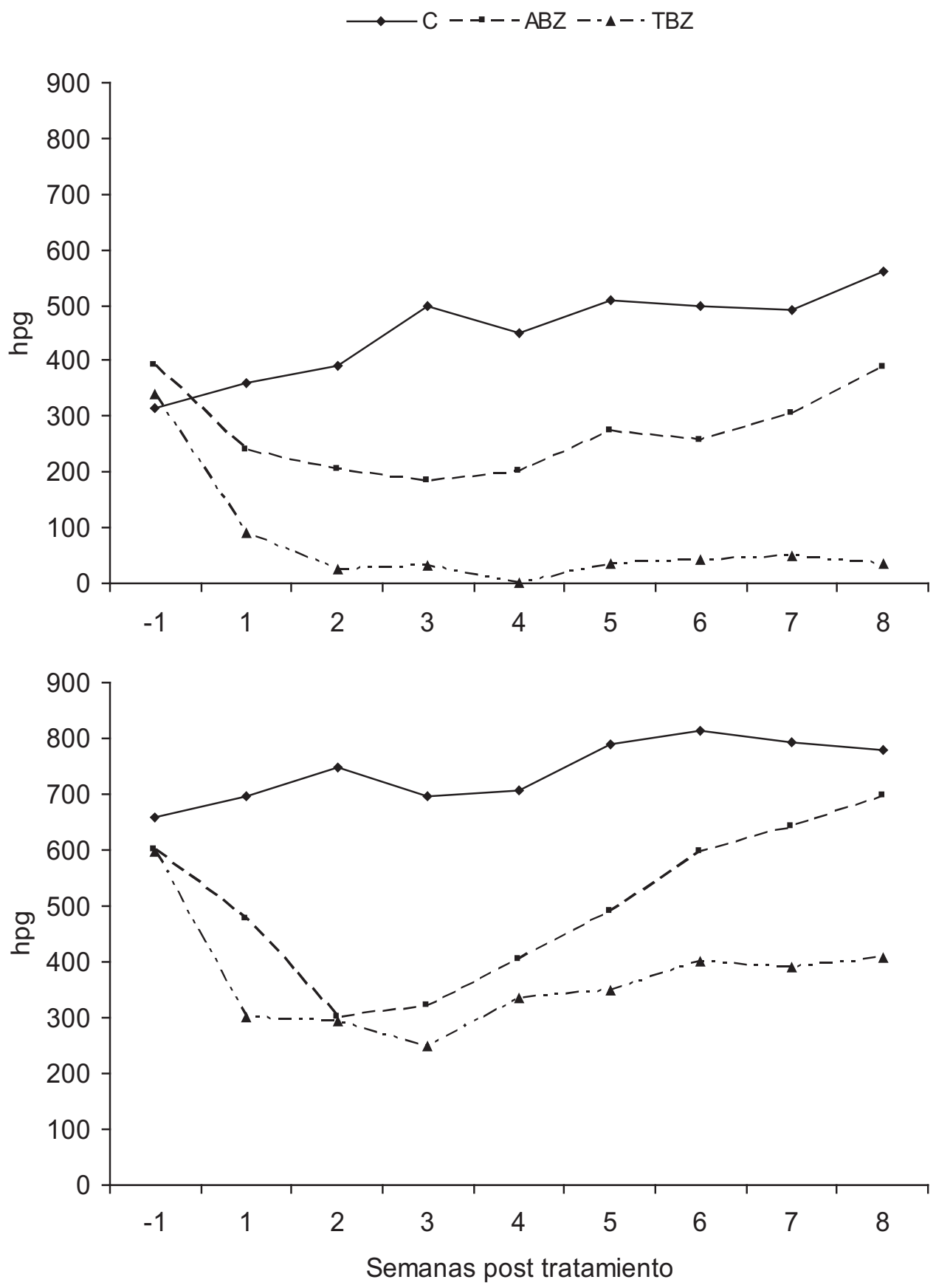

Figura 2. Promedio de huevos de Fasciola hepatica por gramo de heces en ovinos de los grupos control (C), albendazol (ABZ) y triclabendazol (TCBZ) en los rebaños 1 (panel superior) y 2 (panel inferior), desde la semana previa al tratamiento hasta la semana 8 post tratamiento en La Paz, Bolivia

en forma consistente hasta el final del estudio en el rebaño 1 , pero en el rebaño 2 comenzó a aumentar en la semana 7 (Fig. 1).
En el rebaño 1, el tratamiento con triclabendazol permitió obtener más del $90 \%$ de reducción de la oviposición en las sema- 
nas 2 al 8 post tratamiento $(\mathrm{p}<0.05)$, pero en el tratamiento con albendazol se observó una clara resistencia al fármaco dado que la reducción de la oviposición fue menor al 50\% en las semanas siguientes al tratamiento (Fig. 2, panel superior). En el rebaño 2, se notó la resistencia antihelmíntica frente al triclabendazol, donde se obtuvo menos del $65 \%$ de reducción de la oviposición post tratamiento, mientras que el grupo tratado con albendazol, la reducción de la oviposición fue menor a $60 \%$, en las primeras 4 semanas post tratamiento, pero en la semana 7 no hubo diferencia con la oviposición del grupo control (Fig. 2 , panel inferior).

Una oveja del grupo control del rebaño 2 tuvo que ser sacrificada en la semana 7 debido a una fasciolosis aguda grave; no obstante, los datos de este animal fueron considerados, incluyendo el conteo de Fasciolas.

El Cuadro 1 resume el número promedio de fasciolas y el porcentaje de eficacia del antihelmíntico. Se observa la baja eficacia del albendazol en los dos rebaños, en tanto que el triclabendazol fue solamente efectivo en el rebaño 1. Los resultados post mortem mostraron que todos los hígados de los grupos tratados con albendazol en los dos rebaños y triclabendazol en el rebaño 1 presentaron fasciolas en estado adulto y juveniles, en tanto que los hígados de animales tratados con triclabendazol en el rebaño 2 solo presentaron una pequeña cantidad de fasciolas juveniles.

\section{Discusión}

El presente estudio confirma la presencia de cepas resistentes de $F$. hepatica al triclabendazol y al albendazol. Los niveles serológicos de GGT y los hpg en las muestras fecales indican que la infección estuvo presente en los corderos. La actividad de la enzima GGT provee indicación de daño hepático asociados con infección prepatente de F. hepatica (Stojevic et al., 2005).
Los niveles de actividad de la enzima GGT en el grupo control (Fig. 1) se mantuvieron elevados en los dos rebaños, lo cual es un fehaciente indicador de daño hepático provocado por $F$. hepatica (Cordero del Campillo y Rojo, 1999; Meyer y Harvey, 2000; Stojevic et al., 2005). La GGT se produce debido a la proliferación fibrosa de los conductos biliares provocados por los parásitos adultos (Rodríguez, 2005).

La disminución de los niveles de GGT y de hpg en los grupos tratados con triclabendazol se debió a la eliminación de la mayoría de los parásitos adultos y parte de los juveniles de los conductos hepáticos (Gaasenbeek et al., 2001); no obstante, la elevación de estos parámetros en las últimas semanas en el rebaño 2 se debería a la destrucción de los conductos provocada por una nueva generación de fasciolas juveniles (Laverde, 2007) y, por lo tanto, indicando una pérdida de eficacia de triclabendazol contra $F$. hepatica. La eficacia de ABZ contra $F$. hepatica fue reducida, especialmente en el rebaño 2, siendo una muestra de la resistencia obtenida contra este fármaco (Leathwick et al., 2006).

Fairweather y Boray (1999) proponen diferentes estrategias para reducir la velocidad del desarrollo de resistencia antihelmíntica. El tratamiento estratégico con antihelmínticos basado en el conocimiento de epidemiología de la F. hepatica y un manejo adecuado del rebaño puede reducir la infección a un nivel bajo y permite controlar la enfermedad con una baja frecuencia de tratamiento (Boray et al., 1985; Malone, 1997; Márquez, 2003). La reducción de frecuencia del tratamiento también puede reducir la oportunidad del desarrollo de resistencia de droga, sobre todo en áreas de alto riesgo, dónde más tratamientos parecen ser necesarios. En estas áreas, puede ser necesario desarrollar regímenes de tratamientos estratégicos que minimicen la frecuencia del tratamiento y el monitoreo de la eficacia del tratamiento (Mottier y Lanusse, 2001). Obviamente, la prueba de reducción de oviposición solo 
proporciona una indicación de eficacia durante el periodo de patencia y no puede descubrir la resistencia en vías de desarrollo entre las fases inmaduras (Gaasenbeek et al., 2001; Laverde, 2007).

No obstante lo anterior, estudios en Australia han demostrado que el uso combinado de drogas con diferentes modos de acción puede ser eficaz contra las especies resistentes de F. hepatica (Boray, 1997), aunque este posibilidad tiene el riesgo de producir resistencia múltiple a las drogas (Mottier y Lanusse, 2001). Otras alternativas incluyen el uso de vacunas con antígenos potenciales como el glutathione-S-transferase y cathepsin-L1 (Spithill y Dalton, 1998), y el uso de nosodes (Laverde, 2007).

El presente trabajo permite concluir que existe resistencia de $F$. hepatica frente a albendazol en los dos rebaños y que la resistencia al triclabendazol se encuentra en proceso de establecimiento en uno de los rebaños.

\section{Literatura Citada}

1. Álvarez I, Mottier L, Lanusse C. 2007. Drug transfer into target helmint parasites. Trends Parasitol 3: 97-104.

2. Anziani $O$, Zimmermann $G$, Guglielmone A, Vásquez R, Suárez V. 2001. Avermectin resistance in Cooperia pectinata in cattle in Argentina. Vet Rec 149: 58-59.

3. Boray JC, Jackson R, Strong MB. 1985. Chemoprophylaxis of fascioliasis with triclabendazole. NZ Vet J 33: 182185.

4. Boray JC. 1997. Chemotherapy of infections with Fasciolidae. In: Boray JC (ed). Immunology, pathobiology and control of fasciolosis. Rahway, NJ: MSD AGVET. p 83-97.

5. Cordero del Campillo M, Rojo FA. 1999. Parasitologia veterinaria. Madrid: McGraw-Hill Interamericana. 968 p.
6. Fairweather I, Boray JC. 1999. Fasciolicides: efficacy, actions, resistance and its management. Vet J 158: 81-112.

7. Fiel C, Saumel C, Estefan P, Rodríguez E, Salaberry G. 2000. Resistencia de los nematodos Trichostrongylideos -Cooperia y Trichostrongylusa tratamientos con avermectinas en bovinos de la Pampa Húmeda, Argentina. Rev Med Vet 81: 310-315.

8. Gaasenbeek C, Moll L, Cornelissen J, Vellema P, Borgsteede F. 2001. An experimental study on triclabendazole resistance of Fasciola hepatica in sheep. Vet Parasitol 95: 37-43.

9. Jackson F. 1993. Anthelmintic resistance - the state of play. Br Vet $\mathrm{J}$ 149: 123-138.

10. Laverde L. 2007. Efecto del nosodes fasciolinum y del fármaco triclabendazol sobre la oviposición de Fasciola hepática en bovinos. Tesis Maestría. Valdivia: Facultad de Ciencias Veterinarias, Univ Austral de Chile. 78 p.

11. Leathwick D, Miller C, Atkinson D, Haack $N$, Alexander R, Oliver A-M, Waghorn T, Potter J, Sutherland I. 2006. Drenching adult ewes: Implications of anthelmintic treatments pre- and post-lambing on the development of anthelmintic resistance. NZ Vet J 54: 297-304.

12. Malone JB. 1997. The landscape epidemiology of fasciolosis: geographic determinants of disease risk. In: Boray JC (ed). Immunology, pathobiology and control of fasciolosis. Rahway, NJ: MSD AGVET. p 65-81.

13. Márquez D. 2003. Resistencia a los antihelmínticos: Origen, desarrollo y control. Corpoica 1: 55-71.

14. Mason PC, McKay CH. 2006. Field studies investigating anthelmintic resistance in young cattle on five farms in New Zealand. NZ Vet J 54: 318-322.

15. Meyer D, Harvey J. 2000. El laboratorio en Medicina Veterinaria interpretación y diagnóstico. Buenos Aires: Interamericana Ed. 398 p. 
16. Moenen-Locoz AS. 1998. Estudio comparativo de la efectividad de cinco productos comerciales que contienen Ivermectina frente a parásitos gastrointestinales del bovino. Tesis de Médico Veterinario. Valdivia: Facultad de Ciencias Veterinarias, Univ Austral de Chile. 24 p.

17. Moll L, Gaasenbeek G, Vellema P, Borgsteede F. 2000. Resistence of Fasciola hepatica against Triclabendazole in cattle and sheep in Netherlands. Vet Parasitol 91: 153-158.

18. Mottier L, Lanusse C. 2001. Bases moleculares de la resistancia a fármacos antihelmínticos. Med Vet 82: 74-85.

19. Overend D, Bowen F. 1995. Resistance of Fasciola hepatica to Triclabendazole. Austr Vet J 72: 275-276.

20. Rimbaud E, Zúniga P, Doña M, Pineda N, Luna L, Rivera G, et al. 2005. Primer diagnóstico de resistencia a levamisol y lactonas macrocíclicas en nemátodos gastrointestinales parásitos de ovinos en Nicaragua. REDVET 6(5). [Internet]. Disponible en: http:// www.veterinaria.org/revistas/redvet/ n050505/050532.pdf

21. Rodríguez L. 2005. Efecto de la aplicación de fasciolicidas sobre el decomiso de hígado en novillos afectados naturalmente con Fasciola hepática. Tesis de Médico Veterinario. Valdivia: Facultad de Ciencias Veterinarias, Univ Austral de Chile. $26 \mathrm{p}$.

22. Romero J, Boero C, Vásquez R, Aristizábal MT, Baldo A. 1998. Estudio de la resistencia a antihelmínticos en majadas de la mesopotamia argentina. Rev Med Vet 70: 342-346.

23. Ross JG, O'Hagan J. 1966. A biological technique to assess numbers of Fasciola hepatica metacercariae on pasture. J Helminthol 40: 375-378.

24. Sievers G, Alocilla A. 2007. Determinación de resistencia antihelmíntica frente a ivermectina de nematodos del bovino en dos predios del sur de Chile. Arch Med Vet 39: 67-69.
25. Sievers G, Fuentealba C. 2003. Comparación de la efectividad antihelmíntica de seis productos comerciales que contienen lactonas macrocíclicas frente a nematodos gastrointestinales del bovino. Arch Med Vet 35: 81-88.

26. Spithill TW, Dalton JP. 1998. Progress in development of liver fluke vaccines. Parasitol Today 14: 224-228.

27. Stafford K, Coles GC. 1999. Nematode control practices and anthelmintic resistance in dairy calves in the south west of England. Vet Rec 144: 659-661.

28. StojeviaeZ, Piršljin J, Milinkoviae-Tur S, Zdelar-Tuk M, Beer LjubiaeB. 2005. Activities of AST, ALT and GGT in clinically healthy dairy cows during lactation and in the dry period. Veterinarski Arbir 75: 67-73.

29. Suarez VH, Cristel SL. 2006. Anthelmintic resistance in cattle nematode in the western Pampeana Region of Argentina. Vet Parasitol 99: 56-59.

30. Teuscher. 1965. A new single method of examine faeces for the diagnosis of helminth diseases of rumiants. Zbl Vet Med 12: 241-248.

31. Vermunt J, West D, Pomroy W. 1995. Multiple resistance to ivermectin and oxfendazole in Cooperia species of cattle in New Zealand. Vet Rec 143: 443-446.

32. Suarez VH, Cristel SL. 2006. Anthelmintic resistance in cattle nematode in the western Pampeana Region of Argentina. Vet Parasitol 99: 56-59.

33. Teuscher. 1965. A new single method of examine faeces for the diagnosis of helminth diseases of rumiants. Zbl Vet Med 12: 241-248.

34. Vermunt J, West D, Pomroy W. 1995. Multiple resistance to ivermectin and oxfendazole in Cooperia species of cattle in New Zealand. Vet Rec 143: 443-446.

35. Waghorn TS, Leathwick DM, Rhodes AP, Jackson R, Pomroy WE, West DM, Moffat JR. 2006. Prevalence of anthelmintic resistance on 62 beef cattle farms in the North Island of New Zealand. NZ Vet J 54: 278-282. 
36. West DJ, Vermunt J, Pomroy W, Bentall $H$. 1994. Inefficacy of ivermectin againts Cooperia spp infection in cattle. N Z Vet J 42: 192-193.

37. Witzendorff C, Quintana I, Sievers $G$, Schnieder T, Samson-Himmelstjerna G. 2003. Estudio sobre resistencia frente a los bencimidazoles de pequeños estróngilos (Cyathostominae) del equino en el sur de Chile. Arch Med Vet 35(2): 187-194.

38. Young K, Garza V, Snowden K, Dobson RJ, Powel D, Craig TM. 1999. Parasite diversity and anthelmintic resistance in two herds of horses. Vet Parasitol 85: 205-214. 